\title{
Sobre os tipos de Lepidoptera depositados em museus brasileiros. XXV. Nymphalidae (Charaxinae) descrito por Mário Rosa (suplemento); Zygaenidae por A.M. da Costa Lima; Saturniidae (Hemileucinae) por A. Mabilde (suplemento), Castniidae por L. Pfeiffer e Arctiidae (Pericopinae) por Oscar Monte
}

\author{
Olaf H.H. Mielke ${ }^{2}$ \\ Mirna M. Casagrande ${ }^{2}$
}

\begin{abstract}
About the types of Lepidoptera depositated in Brazilian Museums. XXV. Nymphalidae (Charaxinae) described by Mario Rosa (supplement); Zygaenidae by A.M. da Costa Lima; Saturniidae (Hemileucinae) by A. Mabilde (supplement); Castniidae by L. Pfeiffer and Arctiidae (Pericopinae) by Oscar Monte. A list of Nymphalidae (Charaxinae) described by Mário Rosa (supplement); Zygaenidae by A. M. da Costa Lima, Saturniidae (Hemileucinae) by A. Mabilde (supplement), Castniidae by L. Pfeiffer, and Arctiidae (Pericopinae) by O. Monte, with repositories of their types are presented. Lectotypes are designated for Dirphia glauca Mabilde, 1986 and Castnia pellonia catenigra Pfeiffer, 1917.

KEY WORDS. Lepidoptera, types, brazilian museums
\end{abstract}

Com esta série espera-se que os tipos depositados em museus brasileiros, por autores mais antigos, tenham sido identificados. Continuar-se-á procurando por mais informações sobre $o$ assunto.

As espécies descritas por Lauro Travassos, Lauro Travassos Filho, Keith Brown Jr., Olaf H.H. Mielke, Mirna M. Casagrande, Victor Becker e A. Rei do Rego Barros têm nas descrições originais a identificação do material tipo, assim como a instituição onde estão depositados.

A metodologia segue MielKe \& CASAGRANDE (1985).

\section{Tipo de forma descrita por Mário Rosa}

Nymphalidae (Charaxinae)

Agrias narcissus f. clevelandiae Rosa, 1927: 11, 1 fêmea, [holótipo], Colonia Cleveland, Oyapock, Norte do Brasil [Amapá]; Museu Nacional.

O holótipo (Fig. 1) mencionado por Mielke \& Casagrande, 1991: 34, como perdido, foi encontrado no Museu Nacional, Rio de Janeiro, $n^{\circ} 6.612$.

Sinônimo de Agrias narcissus narcissus Staudinger, [1885].

1) Contribuição número 1071 do Departamento de Zoologia, Universidade Federal do Paraná.

2) Departamento de Zoologia, Universidade Federal do Paraná. Caixa Postal 19020 , 81531-990 Curitiba, Paraná, Brasil. Bolsista do CNPq. 

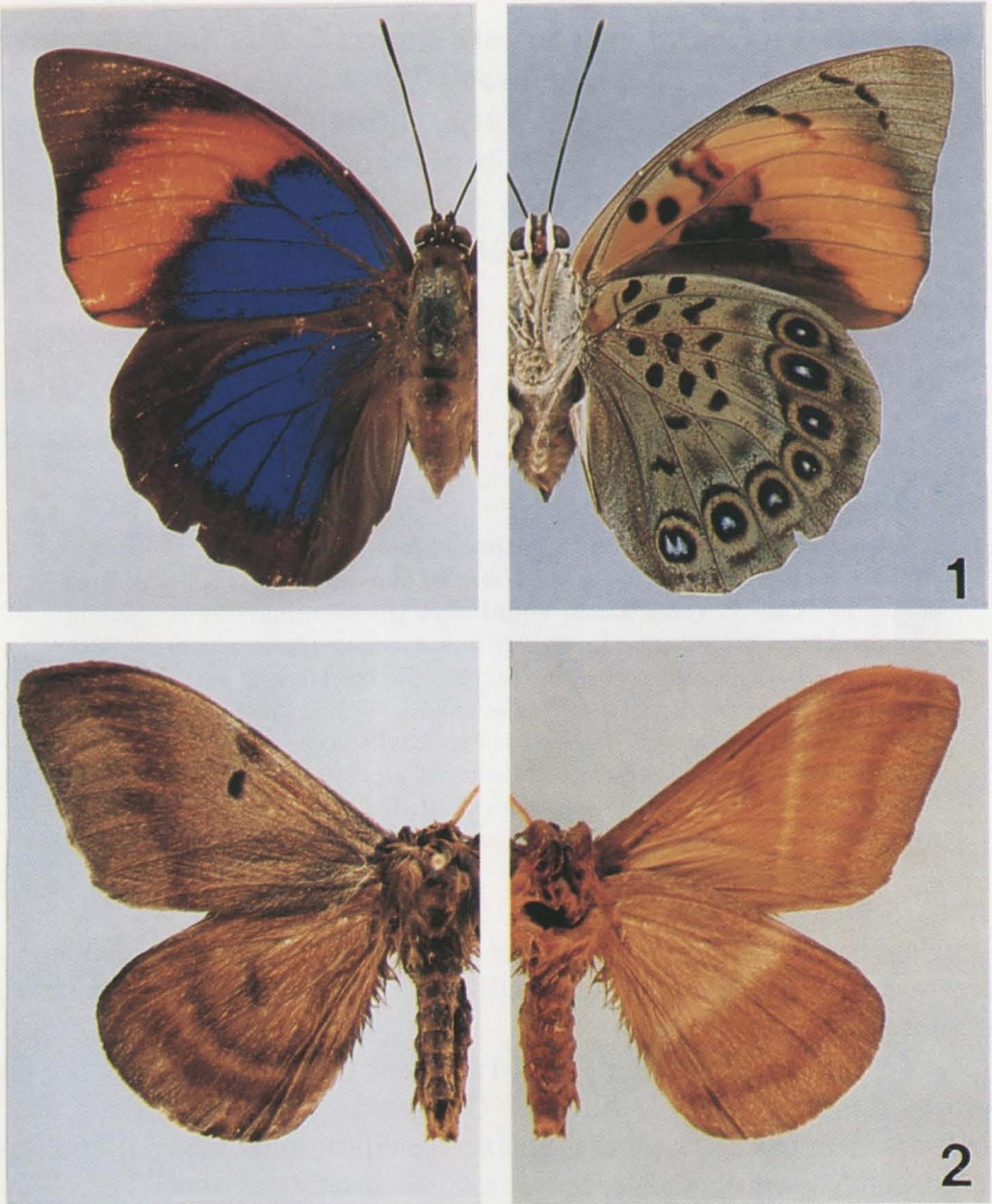

Figs 1-2. (1) Agrias narcissus f. clevelandiae Rosa, holótipo macho, faces dorsal e ventral; (2) Dirphia glauca Mabilde, lectótipo macho, faces dorsal e ventral.

\section{Tipo de espécie descrita por A. M. da Costa Lima}

\section{Zygaenidae}

Stylura brasiliensis Costa Lima, 1928: 25, fig. 1 (venação); Rio de Janeiro, [estado do Rio de Janeiro, Brasil].

$\mathrm{O}(\mathrm{s})$ síntipo(s) deveria(m) estar no Instituto Oswaldo Cruz, Rio de Janeiro, onde o Dr. Costa Lima trabalhou, no entanto, como nada foi encontrado, deve(m) ser considerado(s) perdido(s). 


\section{Tipo de espécie descrita por A. Mabilde}

\section{Saturniidae (Hemileucinae)}

Dirphia glauca Mabilde, 1896: 188, 234, julho, Rio Grande do Sul, [Brasil]; biologia, planta hospedeira.

Os síntipos mencionados por MIELKE \& CASAGRANDE (1990) como perdidos foram encontrados. O macho é aqui designado como lectótipo (Fig. 2) e a fêmea como paralectótipo, ambos estão na Fundação Zoobotânica do Estado do Rio Grande do Sul, Porto Alegre, onde está toda a coleção Mabilde.

Sinônimo sênior de Dirphia baroma (Schaus, 1902). D. glauca foi considerada por LEMAIRE (1996: 45) um nomen oblitum.

\section{Tipos de espécie e subespécie descritas por L. Pfeiffer}

\section{Castniidae}

Castnia intermedia Pfeiffer, 1917: 106, fig. 1 (macho dorsal); 1 macho [holótipo], Rio Negro Superior, leste da Colombia, 800m, Fassl leg., coleção Pfeiffer .

O holótipo (Fig. 3) está no Museu Nacional, Rio de Janeiro, ex coleção Julius Arp, $\mathrm{n}^{\circ} 85.983$. 1995).

Sinônimo de Duboisvalia simulans simulans (Boisduval, [1875]) (LAMAS

Castnia pellonia catenigra Pfeiffer, 1917: 107, fig. 2 (macho, fêmea, dorsal); tipos 1 macho, 1 fêmea, Rio Negro Superior, leste da Colombia, 800m, Fassl leg.; macho na coleção Pfeiffer e fêmea na coleção Fassl.

O tipo macho é aqui designado lectótipo (Fig. 4) e está no Museu Nacional, Rio de Janeiro, ex coleção Julius Arp, n 85.979.

Sinônimo de Duboisvalia ecuadoria truxilla (Westwood, 1877) (LAMAS 1995).

\section{Tipo de espécie descrita por Oscar Monte}

\section{Arctiidae (Pericopinae)}

Pericopsis flavimedia Monte, 1933: 30, 1 fig. (fêmea dorsal), [holó]tipo fềmea, coleção Monte, e uma fêmea no Instituto de Biologia Vegetal, [Rio de Janeiro].

O holótipo (Fig. 5) foi encontrado no Instituto de Biológico, São Paulo, onde parte da coleção O. Monte esta depositada.

Sinônimo de Dysschema hypoxantha Hübner, 1818. 

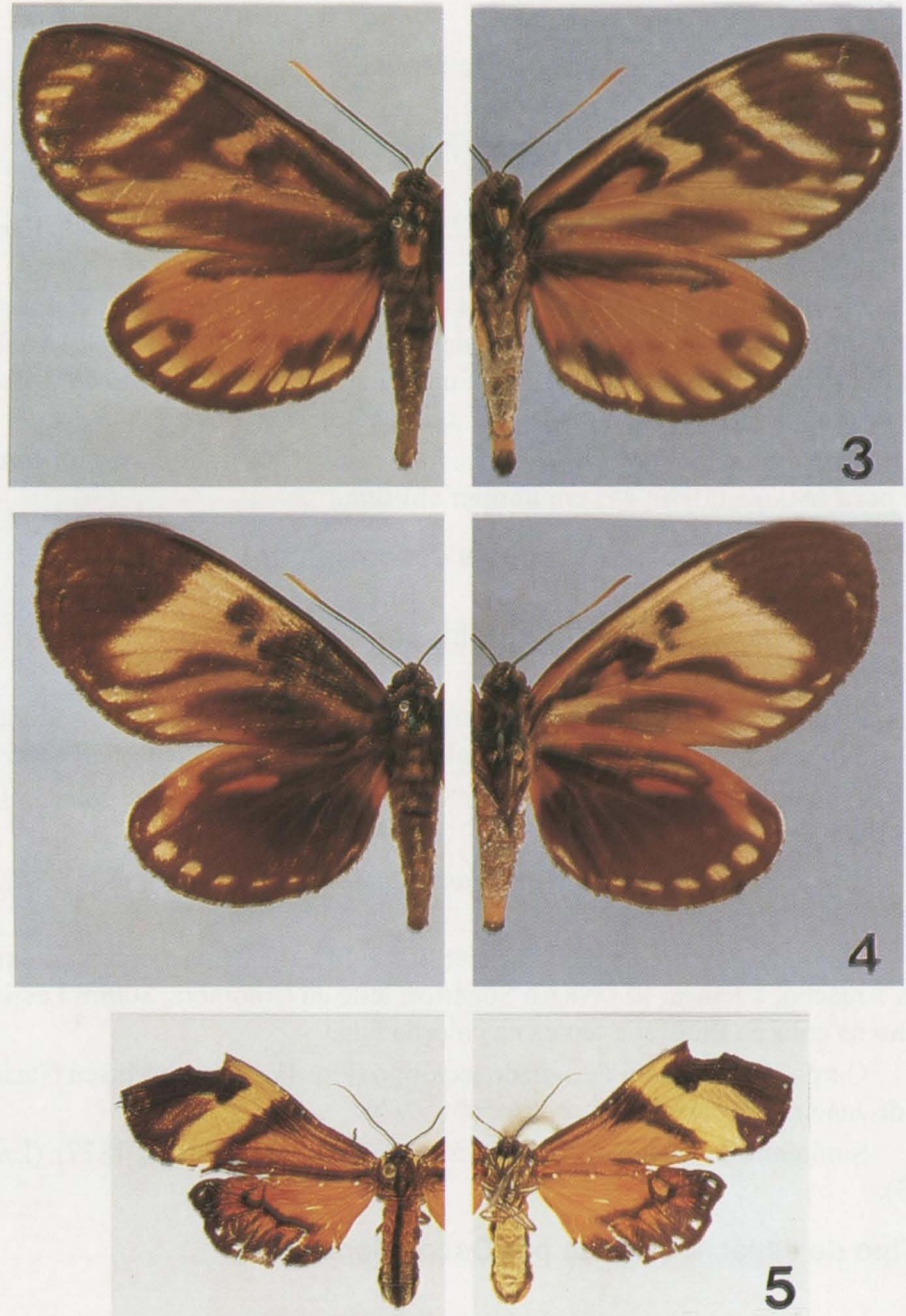

Figs 3-5. (3) Castnia intermedia Pfeiffer, holótipo macho, faces dorsal e ventral; (4) Castnia pellonia catenigra Pfeiffer, lectótipo macho, faces dorsal e ventral; (5) Pericopsis flavimedia Monte, [holó]tipo fêmea, faces dorsal e ventral.

\section{REFERÊNCIAS BIBLIOGRÁFICAS}

Costa Lima, A.M. 1928. Sobre as espécies do gênero "Stylura" Burmeister

(Lepidoptera - Zygaenidae). Bol. Mus. Nac., Rio de Janeiro, 4 (1): 25.

LAMAS, G. 1995. A critical review of J.Y. Miller's Checklist of the Neotropical 
Castniidae (Lepidoptera). Rev. peruana Ent. 37: 73-87.

LEMAIRE, C. 1996. Saturniidae, p.28-49. In: J.B. HEPPNER (Ed.). Atlas of neotropical Lepidoptera. Checklist: Part 4B. Drepanoidea - Bombycoidea Sphingoidea. Gainesville, Scientific Publishers, L+87p.

MABILDE, A. 1896. Borboletas do Estado do Rio Grande do Sul. Guia practica para os principiantes collecccionadores de insectos contendo a descrição fiel de perto de 1000 borboletas com 280 figuras lythographadas em tamanho, formas e desenhos conforme o natural. Estudo sobre a vida de insetos do Rio Grande do Sul e sobre a caça, classificação de uma collecção mais ou menos regular. Porto Alegre, Gundlach \& Schuldt, 238p.

MielKe, O.H.H. \& M.M. CASAgRANDE. 1985. Sobre os tipos de Lepidoptera depositados em museus brasileiros. I. Papilionidae, Riodinidae, Hesperiidae e Arctiidae, descritos por R.F. D'Almeida. Revta bras. Ent. 29 (2): 317-320. 1986. Sobre os tipos de Lepidoptera depositados em museus brasileiros. VI. Castniidae, Cossidae, Sphingidae e Noctuidae, descritos por Benedicto Raymundo [da Silva]. Revta bras. Ent. 30 (2): 245-249. . 1990. Sobre os tipos de Lepidoptera depositados em museus brasileiros. XXI. Papilionidae, Hesperiidae e Pieridae descritos por F. Hoffmann; Nymphalidae (Brassolinae) por L. Travassos \& E. May; Lycaenidae por M. Draudt (suplemento); Papilionidae por W. Niepelt; Noctuidae por C.M. Biezanko e Saturniidae e Apatelodidae por A. Mabilde. Revta bras. Ent. 34 (4): 713-719. . 1991. Sobre os tipos de Lepidoptera depositados em museus brasileiros. XXII. Nymphalidae (Charaxinae) descritos por A.H. Fassl (suplemento) e Nymphalidae (Charaxinae), Papilionidae e Lycaenidae por M. Rosa. Revta bras. Ent. 35 (1): 29-34.

MonTe, O. 1933. Pericopis flavimedia n.sp. Uma nova espécie de Pericopidae. O Campo, Rio de Janeiro, 4 (11): 30-31.

PFEIFFER, L. 1917. Bemerkungen über einige von Herr A.H. Fassl in Columbien gefangene Castnia=, Urania= und Homidiana $=$ Arten. Ent. Ztsch. 30: 106-107, ?? (sep., p.1-14).

Rosa, M. 1927. Lepidópteros do norte do Brasil. Bol. Mus. Nac., Rio de Janeiro, 2 (4): 11-12.

Recebido em 20.XI.1997; aceito em 13.V.1999 